\title{
DIMENSIONADO DE SISTEMA DE ALMACENAMIENTO PARA HIBRIDACIÓN CON FV A PARTIR DE PREDICCIONES PROBABILÍSTICAS DE IRRADIANCIA
}

\author{
E. Pérez, J. González-Barreda, J. Segarra-Tamarit y H. Beltran \\ Departamento de Ingeniería de Sistemas Industriales y Diseño, Universitat Jaume I, \\ Castelló de la Plana, España pereze@uji.es // ORCID: 0000-0001-8466-3941
}

\begin{abstract}
Resumen
En este trabajo se propone una metodología para dimensionar el sistema de almacenamiento de un sistema híbrido con fotovoltaica (FV) para uso doméstico, a partir de predicciones probabilisticas de la producción solar. Se introduce para ello un modelo basado en Deep Learning que, a partir de estimaciones de la irradiancia pasada en el área que rodea la localización objetivo, obtiene las predicciones de distintos percentiles de la producción $F V$. El dimensionamiento se realiza mediante una optimización lineal que utiliza la función cuantil para garantizar, con un cierto nivel de confianza, que se satisface un perfil de demanda tipo. Finalmente, se introducen y discuten resultados en cuanto a la violación de restricciones que se produce con diferentes tamaños del sistema de almacenamiento mostrándose que, cuando este es superior a 3 h a potencia nominal de la instalación $F V$, las restricciones se satisfacen en más del $99 \%$ de las ocasiones.
\end{abstract}

Palabras clave: aplicaciones fotovoltaicas, sistemas de almacenamiento de energía, predicción probabilística, programación lineal.

\section{INTRODUCCIÓN}

A lo largo de los últimos años, la fotovoltaica (FV) se ha consolidado como la primera tecnología renovable en términos de MW instalados al año a nivel mundial, superando así a la energía eólica [8]. De hecho, según se estima en [18], la capacidad FV global instalada a finales de 2020 superaba los 600 GW.

Este importante crecimiento de las instalaciones FV conlleva, no obstante, la aparición de varios desafíos debidos principalmente a la naturaleza estocástica de su producción [9]. Para afrontarlos, existen diferentes enfoques en la literatura. Destacan entre ellos el desarrollo de modelos de predicción de la irradiancia [17] y el uso de sistemas de almacenamiento. Gracias a la progresiva disminución de los precios de estos últimos [12], su presencia junto a instalaciones FV está siendo ca- da vez más habitual. Así, existen propuestas que los combinan tanto para aplicaciones residenciales [4] como para instalaciones a nivel de red [15].

La utilización de sistemas de almacenamiento, y en particular de baterías de iones de litio, para mejorar la producción de plantas FV también ha sido un campo de investigación importante en los últimos años. La mejora productiva se puede obtener de varias formas, dependiendo de los objetivos de la propuesta: suavizado de potencia [10], control de rampa [2], maximización del beneficio económico obtenido [13], entre otros. Del mismo modo, también se ha trabajado bastante en el reto de dimensionar los sistemas de almacenamiento integrados con instalaciones FV [20]. Existen multitud de enfoques en la literatura en este sentido abarcando desde el dimensionado y localización óptima en las redes de distribución $[21,19,14]$ hasta el dimensionado para la producción en firme utilizando modelos de predicción de irradiancia [1].

La introducción de incertidumbre en la gestión de sistemas de almacenamiento mediante restricciones probabilísticas se ha resuelto con anterioridad en la literatura para su aplicación en micorredes $[3,6]$ pero, a conocimiento de los autores, no para instalaciones residenciales que pretendan evitar el consumo desde la red. En este sentido, este trabajo plantea la determinación de las potencias a verter a red así como las de carga-descarga del sistema de almacenamiento de forma que se garantice, en todo momento y con un nivel de confianza adecuado, una potencia disponible en la instalación superior a la demanda de un usuario residencial tipo evitando la saturación del almacenamiento.

El documento se organiza del siguiente modo: en la sección 2 se presenta un modelo de predicción probabilística de la irradiancia basado en redes neuronales, a partir del cual se obtendrá la predicción de producción FV del sistema. La sección 3 está dedicada al planteamiento del problema de optimización que, utilizando el modelo anterior, permitirá dimensionar el almacenamiento requerido por el sistema. En la sección 4 se presentan los resultados obtenidos en cuanto al dimensionado, mostrando el funcionamiento del sistema híbrido en dos días tipo, claro y nublado. Finalmente, en 
la sección 5 se proponen algunas conclusiones y trabajo futuro.

\section{MODELO DE PREDICCIÓN PROBABILÍSTICA DE IRRADIANCIA}

Para generar la predicción de producción de irradiancia, se ha desarrollado un modelo de Deep Learning basado en el presentado en [11] y cuya arquitectura fundamental puede ser observada en la Figura 1. Como se puede ver, esta utiliza como entrada principal estimaciones de irradiancia pasada en el área que rodea la localización objetivo. Estas matrices de irradiancia pasada se obtienen a partir del algoritmo Surface Insolation under Clear and Cloudy Skies (SICCS) [7], un modelo basado en la física y ajustado empíricamente, que utiliza información de satélite. El tiempo transcurrido entre cada una de las matrices es de 15 minutos, que es el mismo con el que se generan las predicciones finales. Las matrices pasan a continuación por una estructura de Deep Learning compuesta por varias capas convolucionales para detectar el movimiento y las características de las nubes. Además, el modelo utiliza otras entradas secundarias como la irradiancia en el exterior de la atmósfera. Esta variable depende de la fecha, la hora y la localización y es independiente del estado de la atmósfera. Por tanto, es posible obtenerla con precisión para cualquier momento, tanto pasado como futuro. Finalmente, la última entrada utilizada son las mediciones pasadas de irradiancia, que se obtienen en la localización objetivo mediante un piranómetro.

La información obtenida por las capas convolucionales, junto con las entradas secundarias, pasa entonces por varias capas densas para obtener la predicción final. En concreto, el modelo tiene tres capas densas para obtener cada una de sus predicciones y replica esta estructura en paralelo para cubrir todo el horizonte de predicción. Cada uno de estos subgrupos está compuesto por tres capas densas de 300, 100 y 20 unidades. Las dos primeras cuentan con activaciones ReLU, mientras que la última utiliza activaciones softmax para obtener la probabilidad de cada uno de los 20 rangos en los que se han discretizado los valores de irradiancia (desde 0 hasta 1000 con escalones de 50). Por tanto, para cada predicción del modelo se obtiene un vector con estas 20 probabilidades, correspondientes a cada nivel de irradiancia, para cada uno de los instantes de tiempo. La selección de los hiperparámetros del modelo se ha llevado a cabo utilizando un año de datos, de los cuales un $80 \%$ se ha destinado al entrenamiento y el otro $20 \%$ a evaluar el desempeño del modelo. Este proceso de entre-
Tabla 1: Comparación de las probabilidades del modelo con los datos reales.

\begin{tabular}{ccccc}
\hline $\operatorname{Pr}\{\hat{x}>x\}$ & $5 \%$ & $10 \%$ & $90 \%$ & $95 \%$ \\
\hline Modelo & $1.3 \%$ & $2.5 \%$ & $97.1 \%$ & $98.2 \%$ \\
\hline
\end{tabular}

namiento se lleva a cabo en una NVIDIA 1050Ti y dura aproximadamente tres horas y media. El entrenamiento se lleva a cabo para minimizar la entropía cruzada y finaliza cuando los resultados empiezan a empeorar para un subconjunto de validación, lo que permite evitar el overfitting.

Las figuras 2 y 3 muestran la producción FV real y diferentes predicciones obtenidas mediante el modelo probabilístico para un día claro y uno nublado, respectivamente. Puede comprobarse como, para los días mostrados, la producción real queda siempre entre los rangos definidos aunque hay otros días en los que el modelo falla en algún instante puntual, como es de esperar por los intervalos de confianza establecidos. Además, es interesante observar como el rango entre las cotas superior e inferior es proporcionalmente mucho menor para el día claro, reflejando la incertidumbre más baja de la predicción en este tipo de situaciones.

Para evaluar el funcionamiento del modelo se han comparado las predicciones de los cuatro percentiles que se van a utilizar posteriormente con datos reales diferentes a los del desarrollo del modelo. La Tabla 1 muestra la cantidad de valores reales que quedan por debajo de cada una de las predicciones obtenidas del modelo. Se observa que los percentiles 5 y 10 del modelo en realidad dejan por debajo menos valores reales de los esperados, con un $1.3 \%$ y un $2.5 \%$ respectivamente. En cambio, las predicciones de los percentiles 90 y 95, dejan por debajo un $97.1 \%$ y un $98.2 \%$, que es un valor superior al esperado.

\section{DIMENSIONADO DEL ALMACENAMIENTO MEDIANTE OPTIMIZACIÓN PROBABILIISTICA}

Una vez se tiene disponible un modelo de predicción probabilística de la producción del sistema $\mathrm{FV}$, se plantea una estrategia de control del sistema híbrido cuyo principal objetivo es satisfacer la demanda a lo largo de todo el día con el sistema de almacenamiento más pequeño que evita la saturación del mismo. No obstante, puesto que no se conoce con total certeza la producción FV, estas restricciones no pueden asegurarse de manera estricta, sino con un nivel de confianza determinado. Así, puede obtenerse la secuencia de potencias de 


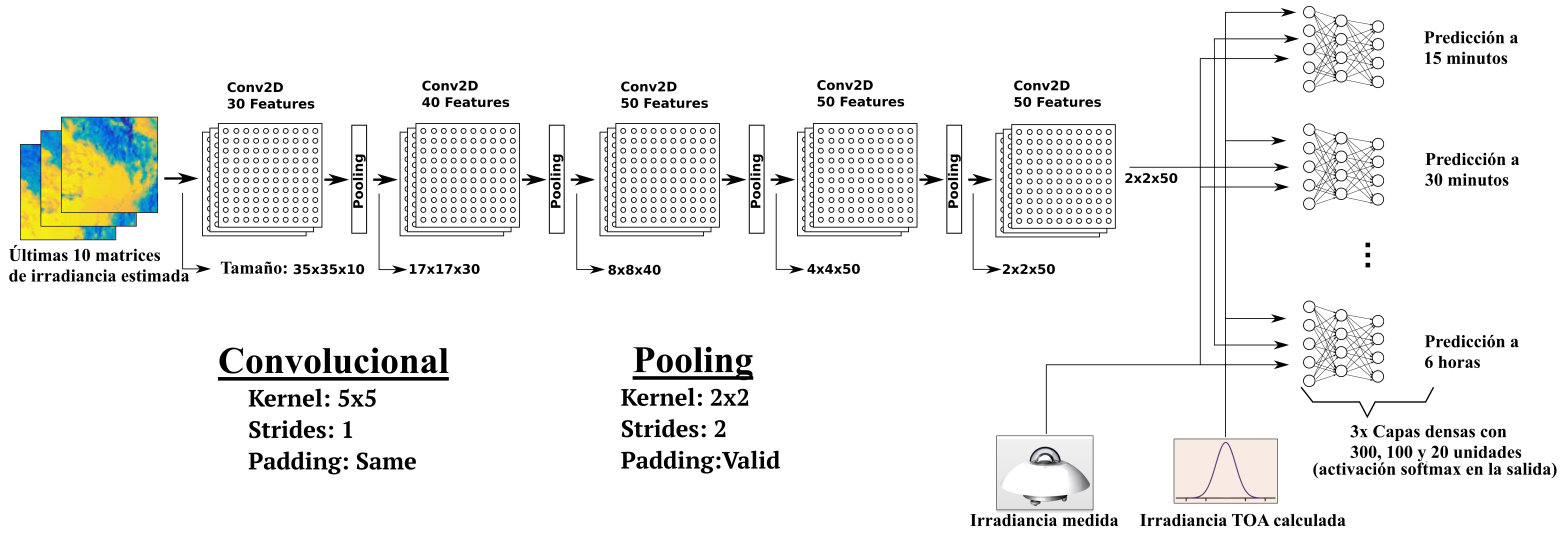

Figura 1: Arquitectura del modelo de Deep Learning con capas convolucionales para detectar el movimiento de las nubes y capas feed forward para hacer predicciones cada 15 minutos.

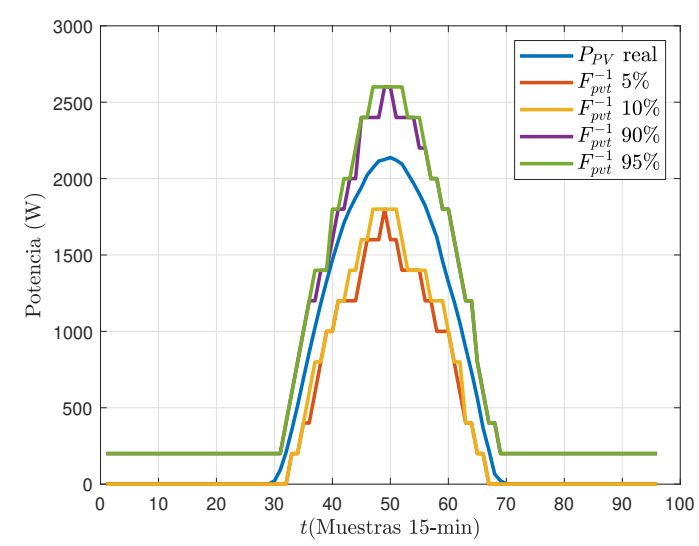

Figura 2: Producción FV real y predicciones probabilísticas para un día claro.

salida del sistema híbrido, $P_{o}$, como:

$$
\begin{aligned}
& \min _{C, P_{o}} C \\
& \text { sujeto } \forall t=1 \ldots N \text { a: } \\
& \quad \operatorname{Pr}\{E(t) \leq C\}>1-\epsilon \\
& \quad \operatorname{Pr}\{E(t) \geq 0\}>1-\epsilon \\
& \quad \operatorname{Pr}\left\{P_{o}(t) \geq P_{d e m}(t)\right\}>1-\epsilon \\
& E(t)=E(t-1)+T \cdot\left(P_{F V}(t)-P_{o}(t)\right)
\end{aligned}
$$

donde $N$ es el número de muestras en un día, 96 cuando se tienen medidas cada 15 minutos; $\operatorname{Pr}\{A\}$ representa la probabilidad del suceso $A ; \epsilon$ es la tolerancia de la condición; $C$ es la capacidad del sistema de almacenamiento; $E(t)$ es la energía almacenada en el mismo en un instante $t ; P_{F V}(t)$ es la potencia producida por los paneles; $P_{o}$ es la potencia de salida del sistema híbrido y $P_{d e m}$ es la potencia demandada por el usuario de la instalación, que quiere garantizarse en todo momento.

La resolución del problema de optimización anterior, tal como está formulado, requeriría que el

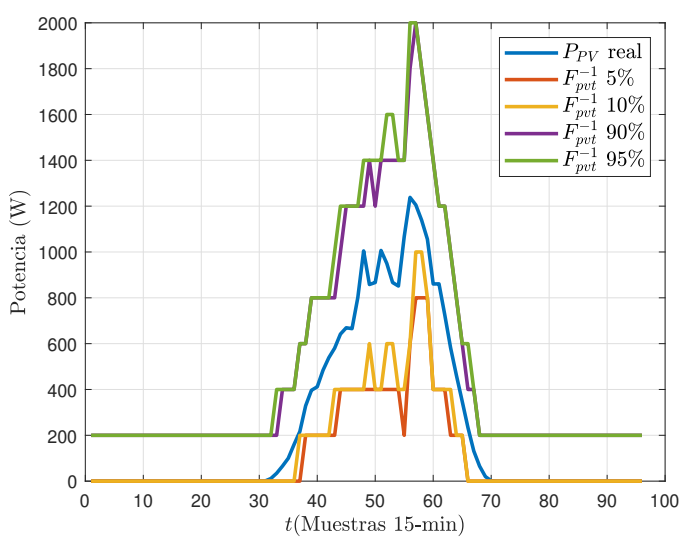

Figura 3: Producción FV real y predicciones probabilísticas para un día nublado.

sistema de almacenamiento fuera capaz de absorber toda la incertidumbre respecto a la producción $\mathrm{FV}$, resultando tamaños de batería muy elevados. No obstante, pueden utilizarse ideas similares a las propuestas en [16] para el control del nivel de embalses. De este modo, puede hacerse uso del hecho de que, en el momento de ajustar la salida de potencia del sistema híbrido, $P_{o}(t)$, se tiene conocimiento de la producción $\mathrm{FV}$ en el instante anterior, $P_{F V}(t-1)$, lo que nos permite definir la potencia de salida como:

$$
P_{0}(t)=P_{F V}(t-1)-h(t)
$$

donde $h(t)$ es una variable de holgura ajustable que determina la diferencia entre ambas potencias.

Substituyendo, se puede trabajar con la ecuación (6) para obtener la evolución de la energía almacenada en la batería. Tomando $P_{F V}(-1)=0 \mathrm{y}$ siendo $E(0)=E_{0}$ la carga inicial de la batería, 
supuesta conocida, se tiene:

$$
\begin{aligned}
E(0)= & E_{0} \\
E(1)= & E_{0}+T\left(P_{F V}(1)-P_{0}(1)\right)= \\
= & E_{0}+T\left(P_{F V}(1)+h(1)\right) \\
E(2)= & E(1)+T\left(P_{F V}(2)-P_{0}(2)\right)= \\
= & E_{0}+T\left(P_{F V}(1)+P_{F V}(2)+h(1)\right. \\
& \left.-P_{F V}(1)+h(2)\right)= \\
= & E_{0}+T\left(P_{F V}(2)+h(1)+h(2)\right) \\
\vdots & \\
E(t)= & E_{0}+T P_{F V}(t)+T \sum_{i=1}^{t} h(i) \\
\vdots & \\
E(N)= & E_{0}+T P_{F V}(N)+T \sum_{i=1}^{N} h(i)
\end{aligned}
$$

Debe recordarse que, en el momento de resolver la optimización, la secuencia de producción FV futura $P_{F V}(t)$ no se conoce de manera determinista, pero se tiene el modelo probabilístico descrito en la sección anterior. Con ello, podemos operar a partir de la ecuación (3):

$$
\operatorname{Pr}\left\{P_{F V}(t) \leq \frac{C-E_{0}}{T}-\sum_{i=1}^{t} h(i)\right\}>1-\epsilon
$$

Y, a partir de esta:

$$
\frac{C-E_{0}}{T}-\sum_{i=1}^{t} h(i)>F_{P f v t}^{-1}(1-\epsilon)
$$

donde $F_{P F V t}^{-1}$ es proporcionada por el susodicho modelo y representa la función de distribución acumulada inversa, o función cuantil, que es el mínimo valor $x$ que garantiza que $\operatorname{Pr}\left\{P_{F V}(t) \leq\right.$ $x\} \geq 1-\epsilon$ :

$$
F_{X}^{-1}(y)=\inf _{x \in \mathbb{R}}\left\{F_{X}(x) \geq y\right\}
$$

De esta forma, la restricción probabilística (3) ha pasado a formularse de manera determinista. Nótese cómo, cuanto menor es el tamaño de la batería $C$, más difícil es que la condición se cumpla. Del mismo modo, un valor elevado de $h(t)$ se traduce en un valor bajo de $P_{o}(t)$ y de la potencia con la que se descarga la batería, lo que dificulta garantizar que la batería no se llene.

En cuanto a la ecuación (4), se puede transformar en una ecuación determinista con un procedimiento similar, recordando que $\operatorname{Pr}\{X \leq x\}+\operatorname{Pr}\{X \geq$ $x\}=1$. De esta forma:

$$
1-\operatorname{Pr}\left\{P_{F V}(t) \leq \frac{-E_{0}}{T}+P_{\text {red }}(t)\right\}>1-\epsilon
$$

O, lo que es lo mismo:

$$
\operatorname{Pr}\left\{P_{F V}(t) \leq \frac{-E_{0}}{T}+P_{\text {red }}(t)\right\}<\epsilon
$$

A partir de esta ecuación podemos aplicar de nuevo la función cuantil para obtener una restricción determinista:

$$
\frac{-E_{0}}{T}+P_{r e d}(t)<F_{P f v}^{-1}(\epsilon)
$$

Análogamente, puede operarse a partir de la ecuación (5):

$$
\begin{array}{r}
1-\operatorname{Pr}\left\{P_{o}(t) \leq P_{d e m}(t)\right\}>1-\epsilon \\
\operatorname{Pr}\left\{P_{F V}(t-1) \leq P_{d e m}(t)+h(t)\right\}<\epsilon \\
h(t)<P_{d e m}(t)+F_{P f v}^{-1}(\epsilon)
\end{array}
$$

Con todo ello, el problema de optimización (1) puede escribirse como:

$$
\begin{gathered}
\operatorname{mín}_{C, P_{o}} C \\
\text { sujeto } \forall t=1 \ldots N \text { a: } \\
\frac{C-E_{0}}{T}-\sum_{i=1}^{t} h(i)>F_{P f v t}^{-1}(1-\epsilon) \\
\frac{-E_{0}}{T}+P_{r e d}(t)<F_{P f v}^{-1}(\epsilon) \\
h(t)<P_{d e m}(t)+F_{P f v}^{-1}(\epsilon)
\end{gathered}
$$

El problema (17) es un problema estándar de programación lineal (LP) para cuya resolución existen múltiples librerías gratuitas.

\section{RESULTADOS Y DISCUSIÓN}

Para analizar el funcionamiento del algoritmo propuesto, se plantea el estudio de un sistema híbrido de FV con almacenamiento para autoconsumo, en el que se quiere conseguir, con el nivel de confianza elegido, que la instalación sea capaz de cubrir en todo momento la demanda y verter los excedentes a la red, cuando estos existan. Se utilizarán datos de irradiancia reales, escalados para una instalación FV de 4 kW, obtenidos en una ubicación mediterránea (Castelló de la plana) durante 187 días (desde el 01/06/2019 hasta el 31/12/2019, excluyendo aquellos días con pérdida de datos por algún motivo). Estos datos no coinciden con los utilizados para entrenar el modelo descrito en la sección 2 , y puede comprobarse que cubren todo tipo de días desde el punto de vista de la irradiancia (desde antes del solsticio de verano hasta después del solsticio de invierno).

En cuanto a la demanda de la instalación, se supondrá que esta se corresponde con un $80 \%$ de la energía total que se prevé producir, en promedio, 


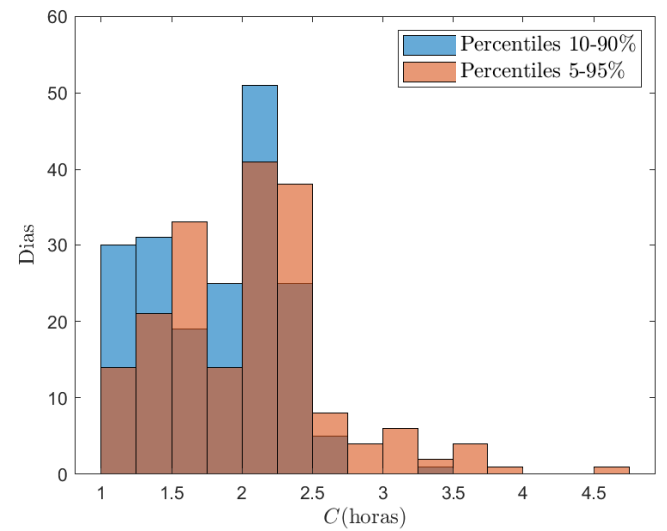

Figura 4: Distribución del tamaño de batería requerido para no saturar en los diferentes días.

mediante la instalación FV durante el día, pero el consumo se distribuirá de acuerdo a un perfil que se extiende a lo largo de las 24 horas. Dicho perfil es el promedio anual de los perfiles de referencia definidos por el Ministerio de Industria para el sector residencial [5], utilizado por las comercializadoras de energía en el Mercado Ibérico de la Electricidad para facturar a los consumidores que no disponen de contador electrónico con telemedida, y por lo tanto se supone que representan un promedio de tendencias. En concreto, se utilizan los perfiles de los clientes con discriminación horaria, que se supone que es el tipo de tarifa más adecuado para sistemas domésticos FV con almacenamiento.

Con todo ello, se lanza la optimización (17) considerando dos intervalos de percentiles diferentes, entre el $5 \%$ y el $95 \%$, y entre el $10 \%$ y el $90 \%$. obteniendo una capacidad diferente para cada uno de los 187 días. En la figura 4 se representa un histograma con los resultados de dichas optimizaciones. Puede comprobarse como, con los percentiles 5\%-95\%, para garantizar que todos los días se cumplan las restricciones impuestas, se necesita una batería de más de 4.5 horas de capacidad a potencia nominal de la instalación FV. No obstante, el tamaño queda determinado por un único día en toda la secuencia. Es más, son muy pocos días los que necesita una batería superior a las 3.5 horas de capacidad. Por otra parte, con los percentiles $10 \%-90 \%$, sólo hay un día que requiere una batería de más de 3.25 horas, y todos los demás garantizan el cumplimiento de restricciones con baterías de 2.75 horas, o inferiores.

Las figuras 5 y 6 muestran, respectivamente la evolución de las potencias del sistema y el estado de carga (state of charge, SoC) de una batería de 14 $\mathrm{kWh}$ de tamaño (equivalente a $3.5 \mathrm{~h}$ ) para los días claro y nublado cuyos percentiles de predicción se muestran en las figuras 2 y 3 , respectivamente.
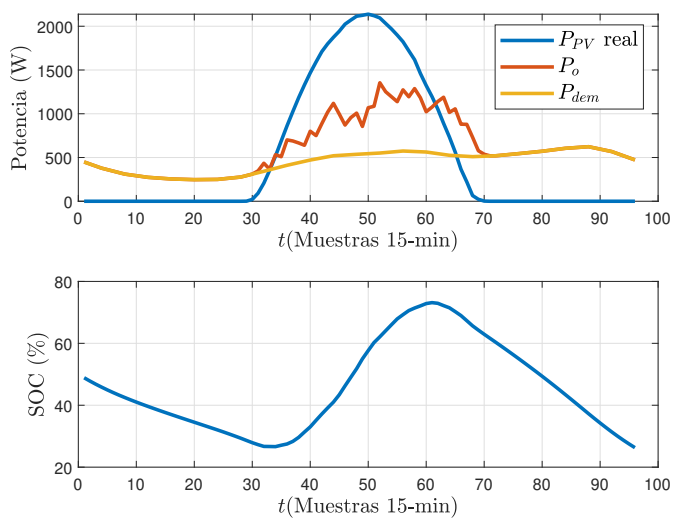

Figura 5: Evolución de potencias del sistema y SoC de la batería, día claro.
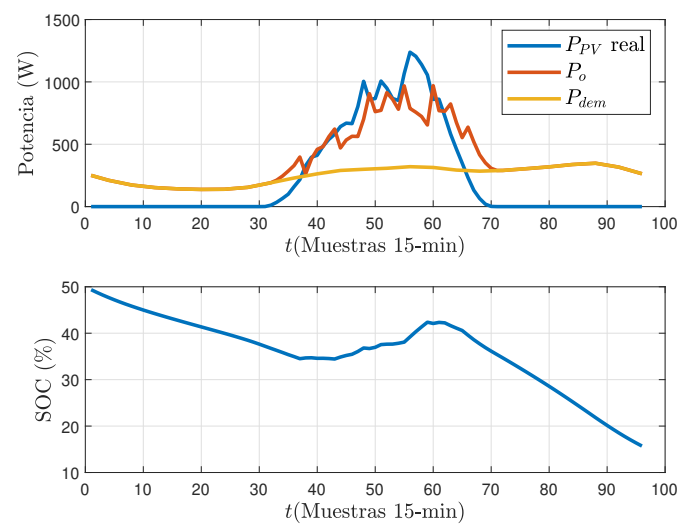

Figura 6: Evolución de potencias del sistema y SoC de la batería, día nublado.

Para ambos días puede comprobarse cómo la optimización decide que el sistema híbrido produzca, durante las horas nocturnas, la mínima potencia suficiente para satisfacer la demanda, a costa de que la batería se descargue. Durante el día, no obstante, el sistema vierte parte de la potencia a la red. Para ambos días puede observarse que, en los tramos en los que la potencia de salida $P_{o}$ se aleja de la curva de demanda $P_{d e m}$, tiene una forma con dientes de sierra. Esto se debe a que, continuamente, el sistema va ajustando la potencia de salida en función de la medida anterior de potencia FV producida, de acuerdo a la ecuación (7). En cuanto a la evolución del SoC, se comprueba que en ninguno de los dos días la batería está cerca de saturarse, por lo que el tamaño de la misma resulta suficiente. Este hecho es también razonable si se revisan las figuras 2 y 3 , en las que puede verse como la producción FV real no se acerca a los extremos marcados por los percentiles sino que sigue una trayectoria bastante central. La saturación de la batería sólo se habría podido producir si la potencia FV hubiera excedido en algún punto dichos percentiles. 
Tabla 2: Violación de restricciones para diferentes tamaños de batería.

\begin{tabular}{ccc}
\hline$C$ & Percent. 5\%-95\% & Percent. 10\%-90\% \\
\hline $1 \mathrm{~h}$ & $44.97 \%$ & $40.91 \%$ \\
$2 \mathrm{~h}$ & $6.73 \%$ & $5.31 \%$ \\
$2.5 \mathrm{~h}$ & $1.60 \%$ & $1.44 \%$ \\
$3 \mathrm{~h}$ & $0.98 \%$ & $1.33 \%$ \\
$3.5 \mathrm{~h}$ & $0.67 \%$ & $1.31 \%$ \\
\hline
\end{tabular}

Los resultados obtenidos para los días anteriores, y algunos otros, hacen sospechar que probablemente el tamaño de batería calculado a partir de la optimización esté algo sobredimensionado durante una gran parte del tiempo de funcionamiento del sistema. Por esta razón, a continuación se calculará, para todos los días de la secuencia, la violación de restricciones que se produce para diferentes tamaños de batería, con los resultados obtenidos por las dos posibles optimizaciones con los percentiles $5 \%-95 \%$, por un lado, y los percentiles 10\%-90\%, por el otro. De este modo, la tabla 2 muestra para qué porcentaje del total de muestras (187 días $\times$ 96 muestras/día) la batería está llena, o vacía, o la potencia de salida $P_{o}$ no es superior a la demanda $P_{d e m}$. Los resultados muestran como, para una batería con una capacidad inferior a las 2.5 horas a potencia nominal de la instalación $\mathrm{FV}$, el nivel de violación de restricciones es probablemente demasiado elevado para ser aceptado. Este resultado es coherente con lo observado en el histograma 4.

Además, cuando la la capacidad de la batería es suficiente, se comprueba que la violación de las restricciones es inferior cuando se utiliza la estrategia de control derivada de la optimización con los percentiles 5\%-95\% que con los percentiles $10 \%$ $90 \%$. Esto es debido a que en el primer caso, la optimización considera condiciones más desfavorables que en el segundo. No obstante, para tamaños de batería inferiores esto no se cumple, porque aplicar el resultado de una optimización pensada con un tamaño de batería considerablemente superior lleva a un mayor grado de suboptimalidad.

\section{CONCLUSIONES Y TRABAJO FUTURO}

En este trabajo se ha propuesto una metodología para dimensionar el sistema de almacenamiento de un sistema híbrido con FV para uso doméstico a partir de predicciones probabilísticas de la producción solar. Dicho dimensionamiento se realiza de modo tal que permita al usuario, con un cierto nivel de confianza, satisfacer un perfil de demanda tipo sin que se produzca la saturación del acumu- lador de energía.

Los resultados de la optimización y de la simulación realizada a partir de datos reales de irradiancia para la ubicación objeto de estudio muestran que, a partir de tamaños del sistema superiores a 3 horas a potencia nominal de la instalación FV, el porcentaje de tiempo en el que se produce la violación de alguna de las restricciones es inferior al $1 \%$ y que, para dichos tamaños, es más conveniente utilizar para la optimización predicciones basada en percentiles 5\%-95\% que 10\%-90\%.

En trabajos posteriores en esta línea, se pretende aplicar el modelo probabilístico desarrollado a sistemas fotovoltaicos de mayor tamaño con participación en diferentes mercados eléctricos y que, por lo tanto, deben obtener la secuencia óptima de potencias con una mayor antelación.

\section{Agradecimientos}

Los autores agradecen el apoyo de la Universitat Jaume I de Castelló mediante su programa Estudia e Investiga en la UJI y a la Generalitat Valenciana y el Fondo Social Europeo por la ayuda concedida con código ACIF/2019/106 en su programa de Subvenciones para la contractación de personal investigador de carácter predoctoral.

\section{English summary SIZING OF ENERGY STORAGE SYSTEM FOR HIBRIDIZATION WITH PV BASED ON PROBABILISTIC FO- RECASTS OF IRRADIANCE}
Abstract
In this work, a methodology is proposed to size the storage system of a hybrid pho- tovoltaic (PV) system for domestic use, based on probabilistic predictions of solar production. To do so, a model based on Deep Learning is introduced which, based on estimates of past irradiance in the area surrounding the target location, obtains the forecasts of different percentiles of $P V$ pro- duction. Sizing is carried out through a linear optimization that uses the quantile function to guarantee, with a certain level of confidence, that a typical demand profi- le is satisfied. Finally, results are introdu- ced and discussed regarding the violation of constraints that occurs with different sizes of the storage system, showing that, when they are greater than $3 \mathrm{~h}$ at the nominal po- wer of the PV installation, the constraints 
are satisfied in more than $99 \%$ of the occasions.

Keywords: PV applications, energy storage systems, probabilistic forecasts, linear programming.

\section{Referencias}

[1] H. Beltran, J. Cardo-Miota, J. SegarraTamarit, and E. Pérez, "Battery size determination for photovoltaic capacity firming using deep learning irradiance forecasts," Journal of Energy Storage, vol. 33, p. 102036, 2021.

[2] H. Beltran, I. Tomas Garcia, J. C. AlfonsoGil, and E. Perez, "Levelized Cost of Storage for Li-Ion Batteries Used in PV Power Plants for Ramp-Rate Control," IEEE Transactions on Energy Conversion, vol. 34, no. 1, pp. 554$561,2019$.

[3] O. Ciftci, M. Mehrtash, F. Safdarian, and A. Kargarian, "Chance-constrained microgrid energy management with flexibility constraints provided by battery storage," in 2019 IEEE Texas Power and Energy Conference (TPEC). IEEE, 2019, pp. 1-6.

[4] K. Darcovich, E. R. Henquin, B. Kenney, I. J. Davidson, N. Saldanha, and I. BeausoleilMorrison, "Higher-capacity lithium ion battery chemistries for improved residential energy storage with micro-cogeneration," $A p$ plied Energy, vol. 111, pp. 853-861, 2013.

[5] Dirección General de Política Energética y Minas-Ministerio para la Transición Ecológica, "Resolución de 21 de diciembre de 2018, por la que se aprueba el perfil de consumo y el método de cálculo a efectos de liquidación de energía," Boletín Oficial del Estado Español (BOE), p. 536-713, 2018.

[6] S. Geng, M. Vrakopoulou, and I. A. Hiskens, "Chance-constrained optimal capacity design for a renewable-only islanded microgrid," Electric Power Systems Research, vol. 189, p. 106564, 2020.

[7] W. Greuell, J. F. Meirink, and P. Wang, "Retrieval and validation of global, direct, and diffuse irradiance derived from SEVIRI satellite observations," Journal of Geophysical Research: Atmospheres, vol. 118, no. 5, pp. 2340-2361, mar 2013.
[8] Janet L. Sawin, Jay Rutovitz, Freyr Sverrisson, Hannah E. Murdock, and Rana Adib, "Renewables 2018, Global Status Report," Tech. Rep., 2018.

[9] F. Katiraei and J. R. Agüero, "Solar PV Integration Challenges," IEEE Power and Energy Magazine, vol. 9, no. 3, pp. 62-71, 2011.

[10] X. Li, D. Hui, and X. Lai, "Battery Energy Storage Station (BESS)-Based Smoothing Control of Photovoltaic (PV) and Wind Power Generation Fluctuations," IEEE Transactions on Sustainable Energy, vol. 4, no. 2, pp. 464-473, 2013.

[11] E. Pérez, J. Pérez, J. Segarra-Tamarit, and H. Beltran, "A deep learning model for intra-day forecasting of solar irradiance using satellite-based estimations in the vicinity of a pv power plant," Solar Energy, vol. 218, pp. 652-660, 2021.

[12] O. Schmidt, S. Melchior, A. Hawkes, and I. Staffell, "Projecting the Future Levelized Cost of Electricity Storage Technologies," Joule, vol. 3, no. 1, pp. 81-100, 2019.

[13] J. Segarra-Tamarit, E. Perez, J. C. AlfonsoGil, C. Arino, N. Aparicio, and H. Beltran, "Optimized management of a residential microgrid using a solar power estimation database," in 2017 IEEE 26th International Symposium on Industrial Electronics (ISIE), 2017, pp. 993-998.

[14] F. R. Segundo Sevilla, D. Parra, N. Wyrsch, M. K. Patel, F. Kienzle, and P. Korba, "Techno-economic analysis of battery storage and curtailment in a distribution grid with high PV penetration," Journal of Energy Storage, vol. 17, pp. 73-83, 2018. [Online]. Available: https://doi.org/10.1016/j.est.2018.02.001

[15] E. Vartiainen, G. Masson, C. Breyer, D. Moser, and E. Román Medina, "Impact of weighted average cost of capital, capital expenditure, and other parameters on future utilityscale PV levelised cost of electricity," Progress in Photovoltaics: Research and Applications, vol. n/a, no. n/a, p. pip.3189, aug 2019.

[16] S. Vedula and P. Mujumdar, Water resources systems: modelling techniques and analysis. Tata McGraw-Hill, 2005.

[17] C. Voyant, G. Notton, S. Kalogirou, M.-L. Nivet, C. Paoli, F. Motte, and A. Fouilloy, "Machine learning methods for solar radiation forecasting: A review," Renewable Energy, vol. 105, pp. 569-582, may 2017. 
[18] A. Whiteman, J. Esparrago, S. Rueda, S. Elsayed, and I. Arkhipova, Renewable Energy Capacity Statistics 2019. Abu Dhabi: IRENA, 2019.

[19] L. A. Wong, V. K. Ramachandaramurthy, S. L. Walker, P. Taylor, and M. J. Sanjari, "Optimal placement and sizing of battery energy storage system for losses reduction using whale optimization algorithm," Journal of Energy Storage, vol. 26, no. August, p. 100892, 2019. [Online]. Available: https://doi.org/10.1016/j.est.2019.100892

[20] Y. Yang, S. Bremner, C. Menictas, and M. Kay, "Battery energy storage system size determination in renewable energy systems: A review," pp. 109-125, aug 2018.

[21] Z. Yuan, W. Wang, H. Wang, and A. Yildizbasi, "A new methodology for optimal location and sizing of battery energy storage system in distribution networks for loss reduction," Journal of Energy Storage, vol. 29, no. January, p. 101368, 2020. [Online]. Available: https://doi.org/10.1016/j.est.2020.101368

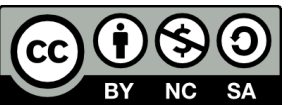

(C) 2021 by the authors. Submitted for possible open access publication under the terms and conditions of the Creative Commons Attribution CC BY-NC-SA 4.0 license (https://creativecommons.org/licenses/by-ncsa/4.0/deed.es). 